\title{
Sobre el empleo del Nomenclátor para el estudio de la distribución espacial de la población en municipios pequeños frente al seccionado censal ${ }^{1}$
}

Nomenclator vs census tracts when studying population spatial distribution in small municipalities

\author{
JuAn José Natera Rivas \\ Universidad de Málaga
}

\author{
Ana Ester Batista Zamora \\ Universidad de Málaga
}

Remedios Larrubia Vargas

Universidad de Málaga

jjnatera@uma.es (ESPAÑA)

Recibido: 08.102019

Aceptado: 07.03.2021

\section{RESUMEN}

El estudio de la distribución de la población en el interior de los municipios rurales presenta importantes dificultades, derivadas de los reducidos volúmenes de población que muchos de ellos presentan, y que, a efectos prácticos, dejan al Padrón Municipal de Habitantes como la única fuente estadística a partir de la cual llevar a cabo este tipo de investigaciones. Desde la vertiente territorial estas dificultades son patentes en lo referente al empleo del seccionado censal, desde el momento en que, debido al escaso número de empadronados, la totalidad del término municipal queda embutida en una única sección, haciendo imposible,

${ }^{1}$ La presente investigación forma parte de los resultados de la Investigación financiada por la Ayuda del Plan Propio de Investigación de la Universidad de Málaga 18-B3-19. 
por tanto, conocer cómo se distribuye la población en su interior. Sin embargo, el empleo del Nomenclátor de Entidades y Núcleos de Población permite soslayar este escollo, por lo que su empleo se muestra como más conveniente en estos casos. Pero no sólo eso, desde el momento en que el Nomenclátor presenta la misma amplitud temática que el Padrón, también es posible no sólo indagar acerca de cómo se distribuye la población en el interior de los municipios, sino también dividirla en grupos a partir de alguna variable relevante, e indagar acerca de la distribución espacial intramunicipal de dichos grupos de población; tareas que no pueden ser llevadas a cabo a partir del seccionado, cuando éste tiene una única sección, y que, incluso siendo posible, hay ocasiones en las que la interpretación de la realidad queda desvirtuada por el trazado de los límites de las secciones. A partir de ejemplos de municipios rurales de la provincia de Málaga, en la presente investigación mostramos alguna de las principales ventajas que el Nomenclátor tiene sobre el seccionado censal. Para ello hemos empleado, por un lado, el volumen de población total, y su distribución entre nacidos en España y nacidos en el extranjero, datos procedentes de la Estadística del Padrón Continuo correspondientes a 2018; por otro, el seccionado censal de ese año, y la cartografía del Nomenclátor, también referida a 2018.

\section{PALABRAS CLAVE}

Nomenclátor; seccionado censal; municipios rurales; distribución espacial de la población; inmigrantes extranjeros.

\section{ABSTRACT}

The study of the spatial distribution of population within rural municipalities faces important difficulties, due to the small volumes of population that many of them present, leaving, at practical purposes, the Padron Municipal de Habitantes as the only statistical source to carry out this type of research. Taking into account the territorial aspect, these difficulties are evident when using census tracts, because when the population number is low, the entire municipal area is embedded in a single one, thus making impossible to know how it is distributed inside the municipality. However, the employment of the Nomenclator de Entidades y Núcleos de Población allows us to avoid this, so it is more convenient. Moreover, since the Nomenclator contains the same number of variables as the Padron, it is also possible not only to inquire about how the population is distributed within the municipalities, but also to divide it into groups using some relevant variable, and describe their intra-municipal spatial distribution. These tasks cannot be carried out using census tracts when only one is delimitated inside the municipality. Moreover, under some circunstances, the interpretation of reality is distorted by the layout of their boundaries. Based on examples of rural municipalities in the province of Malaga, in this paper we show some of the 
main advantages that the Nomenclator has over the census tract. To fullfill this objective, we have used both the total population volume, and its distribution among those born in Spain and born abroad, referred to 2018, and the census tracts and Nomenclator cartography, also referred to 2018.

\section{KEY WORDS}

Nomenclator; census tracts; rural municipalities; population spatial distribution; foreign immigrants.

\section{INTRODUCCIÓN}

El estudio de la población residente en municipios con reducidos volúmenes de residentes ha sido abordado en numerosas investigaciones ${ }^{2}$, no sólo desde el punto de vista de la evolución y caracterización de sus montos (Camarero, 2009; Recaño, 2017), con especial hincapié en el proceso de despoblación rural (Defensor, 2019), sino también desde el significado de la población rural como recurso (Rubio y Pascual, 2017), contando con otras que dan cuenta de su nivel y calidad de vida (Consejo, 2019). También hay disponibles estudios dedicados a los diferentes grupos que pueden identificarse en la población rural, y al posible impacto positivo que la inmigración tendría sobre la dinámica poblacional (Collantes, 2010; Bayona y Gil, 2013).

Sin embargo, son considerablemente más escasos los estudios que abordan la distribución intramunicipal de esta población, más aún aquellos que van un paso más allá y tratan de encontrar diferencias en la población en función del lugar del municipio en el que reside. Este tipo de estudio sí cuenta con un importante corpus cuando los implicados son los municipios urbanos, baste indicar la amplísima bibliografía centrada en la segregación residencial de la población, y no cabe duda de que la disponibilidad de información a nivel inframunicipal en estos municipios urbanos está en la base de este tipo de estudios.

Efectivamente, la disponibilidad real de información - estadística y cartográfica asociada- cuando el objetivo es investigar sobre la distribución espacial de la población en los municipios con pocos empadronados es muy diferente, por escasa, con respecto a cuando el ámbito de estudio es el urbano. Como en el apartado siguiente se muestra, la fuente mayoritariamente empleada en los estudios de población a escala inframunicipal aplicados a la población urbana es la Estadística del Padrón Continuo, con un nivel de desagregación espacial correspondiente a la sección censal. Sin embargo, a efectos prácticos esta misma fuente, a este mismo nivel de desagregación espacial, es inútil cuando queremos trabajar

${ }^{2}$ El corpus bibliográfico referente a cada uno de estos aspectos es extremadamente amplio, por lo que tan sólo ofrecemos algunas referencias recientes seleccionadas a partir de su carácter integrador, referidas al conjunto de España. 
con pequeños (en función de la población) municipios, puesto que debido a su escasa población cuentan con una única sección censal, que equivale al total del municipio; un hecho que, en consecuencia, imposibilita conocer la distribución de la población en el interior del municipio. No obstante, y siendo efectivamente esto así, la información padronal está referida no sólo a las secciones censales, sino también a entidades de población, pudiéndose obtener del Nomenclátor de población. Una fuente que sí permite indagar acerca de cómo se distribuye la población en el interior de estos municipios rurales, y que puede considerarse superior incluso cuando el número de secciones es superior a la unidad.

Esto adquiere especial relevancia cuando los municipios están afectados por dinámicas de población que afectan no sólo a la evolución de los contingentes, sino también a su composición interna y a su distribución territorial. Estos hechos son de gran trascendencia en municipios costeros o cercanos a la línea de costa del litoral mediterráneo, en los que la presencia de lo que se ha venido a denominar inmigrantes residenciales extranjeros ha impactado de manera notable en los volúmenes de población de estos municipios (Collantes et al., 2014). Desde el punto de vista territorial, la mera observación de una realidad en la que progresivamente han venido apareciendo urbanizaciones -unas más legales que otras- en lo que hasta entonces eran entornos netamente rurales (Burriel, 2019), unido a una proliferación desmedida de los diseminados, hacen que la necesidad de una fuente que, siquiera parcialmente, permita acercarse a este fenómeno se resuelva como de gran importancia.

En este contexto debe insertarse el objetivo de los párrafos que siguen, que no es otro que mostrar cómo el empleo del Nomenclátor presenta ventajas con respecto al empleo del seccionado censal cuando se aborda una investigación acerca de la distribución intramunicipal de los residentes en municipios con reducidos volúmenes de población, tomando como ejemplo a la provincia de Málaga. La elección de esta provincia se deriva del hecho de que cuenta con un número importante de municipios de muy pequeña población, pero cercanos a zonas urbanas dinámicas, situados en zonas de montaña media con atractivos naturales y paisajísticos, características que, según Nel-lo (2011) y Hermosilla (1992), en Burriel (2019) son características territoriales que favorecen la aparición de residencias secundarias; además, en estos municipios malagueños se ha venido asentando un importante contingente de población extranjera proveniente de países de la UE -Reino Unido y Alemania, en gran medida-, que añade complejidad tanto a la evolución de los contingentes de residentes, como a sus pautas de distribución territorial.

Para dar cuenta de nuestro objetivo hemos dividido la contribución en 5 apartados. En el primero de ellos repasamos las diferentes opciones de las que disponemos a la hora de abordar un estudio como el que acabamos de indicar. En el segundo indicamos las fuentes, estadísticas y cartográficas, empleadas en esta investigación. El tercero es el núcleo del trabajo, y en él se muestra, a través de ejemplos concretos, cómo efectivamente la distribución espacial correspondiente al Nomenclátor es mejor opción que la correspondiente al seccionado censal a la 
hora de abordar la distribución intramunicipal en pequeños municipios. Conclusiones y bibliografía completan la contribución.

\section{FUENTES PARA EL ESTUDIO DE LA DISTRIBUCIÓN INTRAMUNICIPAL DE LA POBLACIÓN}

A la hora de abordar un estudio acerca de la distribución o la diferenciación residencial intramunicipal de la población, ya sea en ámbitos rurales o urbanos, es necesario, en primer lugar, contar con información estadística que resulte de utilidad desde el punto de vista temático: si lo que se desea es sólo acercarse a cómo se distribuye la población en el interior del municipio, será suficiente con disponer de los totales de población. Pero si, además, se quiere indagar si determinadas características de la población varían en función de su lugar de residencia, o si se quiere indagar acerca de los niveles de diferenciación residencial de grupos de población identificados a partir de una variable o de un conjunto de ellas, entonces la fuente deberá proveernos de dicha información. Ejemplo de este tipo de estudio sería describir la distribución de la población en el interior de un municipio según su edad, o bien según su nacionalidad, etc., como también los estudios de ecología factorial o de segregación urbana. En segundo lugar, también es necesario que la información estadística anterior esté referida a unidades espaciales inframunicipales, de manera que no sólo se sea capaz de caracterizar a la población que reside en el conjunto del municipio, sino que también podamos localizarla a partir de su lugar de residencia. Dos son, por tanto, los tipos de información necesarios: estadística y cartográfica, ambas referidas a las mismas unidades espaciales inframunicipales.

Las fuentes más empleadas en los estudios orientados a la distribución intramunicipal de la población son el Censo y el Padrón Municipal de Habitantes, puesto que son las únicas que nos proveen de la información necesaria para la totalidad del territorio. No obstante, ambas tienen sus pros y sus contras.

Como es sabido, el Censo de Población se realiza cada 10 años, conteniendo una extensísima nómina de variables referidas no sólo a la población, sino también a las viviendas que ocupan y a los hogares que conforman. Sin embargo, el último Censo disponible, de 2011, fue implementado a partir de una metodología muestral (la población objeto de estudio es una muestra del 9\% del conjunto de residentes habituales, en viviendas familiares convencionales o en alojamientos, más aquella otra residente en establecimientos colectivos -INE, 2011-), de forma que, en principio, la enorme amplitud temática disponible queda drásticamente disminuida cuanto más reducida -en términos de población, no de superficie- es la unidad espacial para la cual deseamos obtener la información. A modo de ejemplo, la correspondiente a la distribución por sexo de los ocupados mayores de 16 años según la rama de actividad del establecimiento en el que trabajan sólo está disponible para municipios de más de 16.000 habitantes; o, un caso más extremo, para municipios muy pequeños no es posible construir una simple pirámide de población, desde el momento en que la distribución por edad y sexo en 
cohortes quinquenales no está disponible en su totalidad; hay cohortes para las que no se dispone de información al ser la base poblacional demasiado reducida como para estimar los valores, a partir de las unidades muestrales disponibles en su interior.

El hecho de que cuando más pequeña sea la base poblacional de la unidad espacial para la cual queremos obtener la información censal menor sea su disponibilidad real, impacta de manera decisiva en la utilidad efectiva del Censo a la hora de abordar un estudio de la población a nivel inframunicipal. Esto es así porque las secciones censales -unidad espacial mínima para la que está disponible la información censal- cuentan en su interior con volúmenes de población reducidos, de manera que la posibilidad de rescatar variables relevantes para el estudio de la población se reduce sobremanera, lo que, de facto, descarta al Censo de 2011 como fuente para este tipo de estudio.

La segunda fuente susceptible de ser empleada es el Padrón Municipal de Habitantes. Contamos, a partir de su Estadística Continua, con información anual detallada referida al 1 de enero de cada año. La fuente contiene a la totalidad de la población empadronada en cada uno de los municipios, al derivarse de un recuento y no de un muestreo. Es por ello que, a diferencia del Censo, podemos disponer de la información de cada una de las variables disponibles en el Padrón tanto a nivel municipal como intramunicipal. Sin embargo, su amplitud temática quedó ampliamente recortada desde 1996, conteniendo en la actualidad únicamente información acerca de la estructura por edad y sexo, la nacionalidad, el lugar de nacimiento de los empadronados, y la relación entre lugar de residencia y lugar de nacimiento. En este sentido, se ha indicado que, efectivamente el Padrón presenta una serie de problemas relacionados con el registro efectivo de la población extranjera, especialmente para la población extranjera retirada (Rodríguez, Lardiés y Rodríguez, 2010) cuya presencia en Málaga es considerable. Pero siendo efectivamente esto así, el hecho es que el Padrón es la única fuente que contiene a nivel municipal información acerca de la nacionalidad, el lugar de nacimiento y la distribución por edad de la población empadronada a 1 de enero de cada año, lo que lo convierte en una fuente insustituible para el tipo de estudio al que venimos haciendo referencia.

El segundo tipo de información que necesitamos es la cartográfica, en concreto aquella que contempla la existencia de unidades espaciales intramunicipales: cartografía que, además, debe ser susceptible de ser asociada a la información estadística proveniente del Padrón -dados los problemas que el Censo de Población presenta-.

Cuando el nivel de desagregación espacial es el intramunicipal la información padronal suele presentarse por secciones censales. Estas secciones censales -o electorales- surgen por la necesidad de dividir los municipios de forma rigurosa para la realización del Censo Electoral (Villarín y Segura, 2015: 434), indicando la Ley Orgánica del Régimen Electoral General (LOREG) en su artículo 23, que el número de electores presentes en cada sección censal oscilará entre un mínimo de 500 y un máximo de 2.000, contando cada término municipal con al menos una sección (BOE, 1985). En este sentido, una sección censal puede 
definirse como «Una partición del término municipal caracterizada por: estar preferentemente definida mediante límites fácilmente identificables, tales como accidentes naturales del terreno, construcciones de carácter permanente y viales. Y tener un tamaño entre 1.000 y 2.500 residentes, excepto en el caso que el municipio completo tenga una población inferior» (Eustat, s.f.). En cuanto a los límites físicos, el artículo 24 de la citada ley indica que "las Delegaciones Provinciales de la Oficina del Censo Electoral determinarán [...] los límites de las Secciones Electorales" (BOE, 1985). Por tanto, la sección censal es una unidad administrativa que no necesariamente tiene porqué adecuarse a las necesidades del investigador, reflejo de una situación de la que ya Peter Hagget daba cuenta en los años setenta, cuando indicaba que los datos son transmitidos en "haces", esto es, en áreas administrativas inadecuadas y anacrónicas (Hagget, 1976:243). En este sentido, Ocaña (2005) ofrece una completa revisión de la cuestión del empleo de las secciones censales en estudios de microanálisis social urbano, alguna de cuyas consideraciones podrían extrapolarse también a las investigaciones sobre ámbitos rurales.

Una revisión de la cartografía censal nos muestra que existe una gran disparidad en el tamaño físico que las secciones presentan -más reducido cuanto mayor sea la densidad de población-, pero, además, el hecho de que su definición contemple unos mínimos y máximos de habitantes implica que su estabilidad temporal no es necesariamente tal. Efectivamente, basta una mirada a los registros correspondientes a varios años del Padrón municipal de habitantes para comprobar cómo el número de secciones cambia, por lo general por adición en las periferias más dinámicas de los espacios construidos (vía fragmentación de una sección preexistente), por sustracción en las centrales en las que el volumen de población declina (vía unión de dos o más secciones preexistentes). Pero más allá de estos cambios, que influirían decisivamente en un estudio de corte longitudinal, en nuestro caso nos interesa más el hecho de que cuando los municipios tienen poblaciones muy pequeñas nos encontramos con que cuentan con una única sección censal -recordemos que existe un mínimo de población en su delimitación-, que, desde el punto de vista territorial, equivale a la totalidad del municipio. Así las cosas, en estos casos es imposible realizar cualquier estudio intramunicipal tomando la sección censal como unidad espacial de referencia. Una situación que afecta a la posibilidad de realizar un estudio intramunicipal basada en cualquier variable cuyo nivel de desagregación espacial sea la sección censal, como por ejemplo el interesantísimo Atlas de Distribución de la Renta de los Hogares que el INE ofrece en el marco de la Estadística Experimental.

Sin embargo, además del seccionado censal hay otra división espacial del territorio, que lo cubre de manera exhaustiva, y a la que también queda referida la información padronal: el Nomenclátor de Entidades y Núcleos de población. Aunque es una delimitación sin carácter oficial, pero sí con una gran tradición, el Nomenclátor contiene una nómina de todas las entidades, núcleos de población y diseminados existentes en un municipio. Una entidad singular de población se define como «cualquier área habitable del término municipal, habitada o excepcionalmente deshabitada, claramente diferenciada dentro del mismo, y que es 
conocida por una denominación específica que la identifica sin posibilidad de confusión» (IECA, s.f. 2). Por su parte, un núcleo es «un conjunto de al menos diez edificaciones, que están formando calles, plazas y otras vías urbanas» (Ibid., 2). Todos los municipios están compuestos al menos por una entidad singular de población, entidad que puede tener uno o varios núcleos, o, en ausencia de éstos, ser considerada en su totalidad como diseminado. Así las cosas, el Nomenclátor distribuirá, en el peor de los casos, a la población empadronada en un núcleo y un diseminado, esto es, un escalón más que el que puede obtenerse empleando el seccionado censal. Además el Nomenclátor comparte con éste el que un empadronado no puede ser adscrito a dos unidades espaciales simultáneamente, desde el momento en que toda edificación no integrada en un núcleo pertenece a un diseminado, y una misma vivienda -y, por extensión un mismo empadronado-, no puede pertenecer a dos o más núcleos o a un núcleo y un diseminado a la vez. Pero pese a la utilidad potencial de esta fuente que puede intuirse, el Nomenclátor ha sido empleado en muy escasas ocasiones en investigaciones aplicadas a ámbitos urbanos (Montosa y Reyes, 2017), o rurales. Entre éstas últimas, Natera lo utilizó para dar cuenta de las variaciones en la distribución espacial de la población en tres pequeños municipios de la Costa del Sol (Natera, 1996), y Natera y Batista (2018) lo han utilizado recientemente para investigar la evolución de los volúmenes de población extranjera, y su distribución espacial, en municipios rurales de la comarca malagueña de la Axarquía.

En la web del Instituto Nacional de Estadística -INE- (www.ine.es) es posible consultar la información padronal a este nivel de desagregación espacial, pudiéndose obtener información sobre la distribución por edad y sexo de la población, su nacionalidad y su lugar de nacimiento. Además, en el caso de la Comunidad Autónoma de Andalucía el Instituto de Estadística y Cartografía de Andalucía (IECA) cuenta con una base cartográfica en la que quedan representadas la totalidad de las entidades, núcleos y diseminados existentes en los municipios andaluces, base cartográfica a la que es posible añadir la información estadística padronal obtenida del INE. Como anteriormente hemos indicado, en el caso de los municipios con volúmenes de población muy reducidos, el Nomenclátor la distribuye como mínimo en población en núcleo y en población en diseminado. Pero en una cantidad significativa de ocasiones, en estos municipios pequeños, rurales, la nómina de unidades espaciales a las que el Nomenclátor refiere la población es considerablemente mayor que la correspondiente al seccionado censal. Y a partir de aquí es donde se aprecia la ventaja de emplear el Nomenclátor como fuente para el estudio de la distribución intramunicipal de la población en los municipios de población reducida. En este sentido, y aunque nosotros hemos empleado la base cartográfica desarrollada por el IECA, debemos indicar que el Instituto Geográfico Nacional (www.ign.es) ha desarrollado recientemente la denominada Información Geográfica de Referencia de Poblaciones. En esta base espacial se identifican, entre otros, los núcleos de las Entidades de población, tal y como los define el Nomenclátor, por lo que estimamos que puede ser gran utilidad a la hora de extrapolar nuestros hallazgos al resto de España, desde el momento en que esta base de datos espacial permite hasta cierto punto replicar 
nuestra investigación en aquellas Comunidades que no cuentan con una delimitación espacial propia de las entidades de población.

En este sentido, es necesario indicar que la base espacial el IGN identifica los núcleos de población con mayor precisión de lo que lo hace el IECA, tal y como se aprecia en la figura 1. En ella representamos la delimitación de los núcleos del municipio malagueño de Alcaucín- realizada por el IGN y el IECA, en la que se aprecia que el número de núcleos es el mismo, pero la delimitación es, efectivamente, más precisa en la primera con respecto a la segunda. De cualquier manera, y como venimos indicando, en nuestro ejemplo en ambos casos los núcleos comparten códigos de identificación con el Nomenclátor, lo que permite adscribirles de manera individual, y sin posibilidad de error, la información estadística contenida en éste.

\section{Figura 1. Municipio de Alcaucín (Málaga). Delimitación de núcleos en el IGR Poblaciones y delimitación de Entidades de Población en DERA- IECA-.}
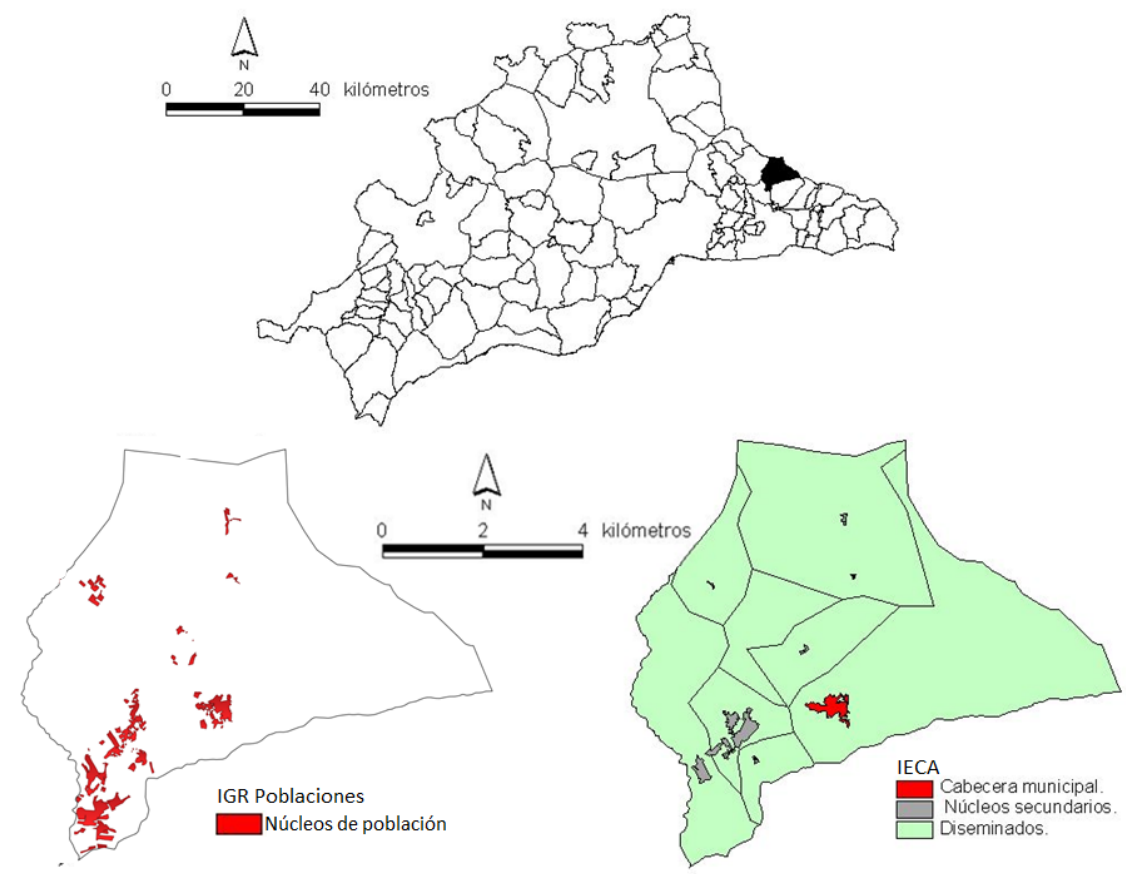

Fuente: Información Geográfica de Referencia -IGR- Poblaciones, IGN, y Datos Espaciales de Referencia de Andalucía -DERA-, IECA. Elaboración propia. 
Sin embargo, en relación con los diseminados, hay que señalar que, a diferencia de la base cartográfica de los Datos Espaciales de Referencia de Andalucía, la Información Geográfica de Poblaciones no delimita los diseminados (figura 1); en consecuencia, toda la información estadística correspondiente a ellos -en los casos en los que el Nomenclátor identificase más de uno- quedaría agrupada en una única entidad de este tipo. De cualquier manera, y tal como indica el propio IGN, la versión disponible en el momento de escribir estas líneas es una BETA, al estar basada en procedimientos automáticos que actualmente están en proceso de revisión y verificación, por lo que cabe esperar cambios en las sucesivas versiones.

Para terminar este apartado, indicaremos que hay aún algunas otras opciones que podrían emplearse para realizar un estudio intramunicipal sobre la distribución de la población. Efectivamente, el IECA pone a disposición de los investigadores datos de población -que incluyen las principales nacionalidadesreferidos a una malla de cuadrículas de $1 \mathrm{~km}^{2}$, y a otra de 250 metros de lado, a través del recurso denominado Distribución Espacial de la Población en Andalucía. En él puede encontrarse la información padronal -junto a otras referentes a características económicas de la población- georeferenciada a estas cuadrículas; sin embargo, el hecho de que haya una cantidad muy significativa de éstas en las que la información no está disponible debido a la preservación del secreto estadístico invalida esta escala. Para ilustrar esta cuestión, tomaremos la información que ofrecemos en la tabla 1: en ella mostramos la situación correspondiente a 5 municipios que hemos empleado como ejemplos en las páginas que siguen: Alcaucín, Benahavís, Ojén, Sedella y Cómpeta. De todas las variables que nos ofrece la fuente, tan sólo nos centraremos en las correspondientes a la estructura y al origen de la población, un total de 16. Es importante indicar que estas mismas 16 variables están disponibles en la Estadística del Padrón Continuo, o pueden construirse a partir de ella, para la totalidad de las unidades espaciales a las que se adscriben -ya sean secciones censales o entidades de población-. Sin embargo, y como inmediatamente vamos a ver, no ocurre lo mismo en el caso de las mallas, puesto que la unidad espacial es mucho menor, y, en consecuencia, la posibilidad de vulneración del secreto estadístico se multiplica.

En la tabla ofrecemos, en primer lugar, el número de cuadrículas correspondientes a cada municipio en 2018; en segundo, el número potencial de valores de los que podríamos disponer de no estar ninguno de ellos afectado por el secreto estadístico (resultante de multiplicar el número de cuadrículas por las 16 variables); por último, el número de casos protegidos, más el porcentaje que suponen sobre el total potencial. Pues bien, en el mejor de los casos, correspondiente al municipio de Cómpeta, el 55,59\% de los valores están protegidos por el secreto estadístico, cifra que asciende al $88,14 \%$ en Sedella, lo que, como habíamos indicado al comienzo del párrafo, invalida en la práctica esta fuente de información. 
Tabla 1. Número de cuadrículas de $250 \times 250$, número de potencial de casos y casos protegidos por el secreto estadístico en municipios seleccionados (2018).

\begin{tabular}{|c|c|c|c|}
\hline Municipio & $\begin{array}{l}\mathrm{N}^{o} \text { de cuadrí- } \\
\text { culas }\end{array}$ & $\mathrm{N}^{o}$ potencial de casos & $\begin{array}{l}\text { Secreto estadís- } \\
\text { tico }\end{array}$ \\
\hline Alcaucín & 51 & 816 & $527(64,58 \%)$ \\
\hline Benahavís & 82 & 1.312 & $963(73,39 \%)$ \\
\hline Cómpeta & 43 & 688 & $385(55,95 \%)$ \\
\hline Ojén & 24 & 384 & $266(69,27 \%)$ \\
\hline Sedella & 29 & 464 & $409(88,14 \%)$ \\
\hline
\end{tabular}

Fuente: Distribución Espacial de la Población en Andalucía (2018). Elaboración propia.

Y otra opción es la delimitación de barrios, unidad alternativa a la sección censal a la que puede quedar referida la información padronal. Pero tan sólo hay un pequeño número de municipios -todos urbanos- que cuentan con esta delimitación espacial, y aún son menos (en el caso de la provincia de Málaga, tan sólo el municipio capital) que cuentan con información estadística referida a ellos, por lo que su aplicabilidad a ámbitos rurales es nula.

Para la ejemplificación de las ventajas que el empleo del Nomenclátor tiene sobre el seccionado censal en el estudio de la distribución de la población en el interior de los municipios rurales hemos empleado las siguientes fuentes estadísticas y cartográficas. En primer lugar hemos obtenido directamente de la página web del INE (www.ine.es) información de la Estadística del Padrón Continuo correspondiente a 2018, el último año disponible con datos definitivos en el momento de escribir estas líneas: en concreto, y para cada uno de los 104 municipios malagueños (y las 1.061 secciones censales en las que se dividen), hemos extraído los totales de población y su distribución según lugar de nacimiento (España o extranjero), un total de 3.201 datos. La elección de ésta variable precisa de una aclaración. Pese a la escasa amplitud temática que nos ofrece el Padrón, sí incluye variables que permiten la cuantificación y el estudio de la población extranjera, a partir de dos variables: nacionalidad y lugar de nacimiento, muy similares, pero no equivalentes. La primera de ellas hace referencia a la nacionalidad del empadronado, que no necesariamente tiene por qué ser siempre la misma, pues puede modificarse en un acto administrativo; la segunda sí es invariable, y no se modifica aun cuando el individuo haya cambiado su nacionalidad. Es así que la consideración de extranjero a partir de su lugar de nacimiento permite obviar el impacto que las nacionalizaciones tienen sobre el volumen total de extranjeros identificados en un municipio determinado. Un impacto que en el caso de determinadas nacionalidades -latinoamericanas- es más que nota- 
ble, y que es preciso tener en cuenta cuando estudiamos la evolución temporal de estos contingentes.

Junto a la información de la Estadística del Padrón Continuo, hemos accedido a la correspondiente al Nomenclátor de 2018, esta vez a partir de un procesamiento especial solicitado al $\mathrm{INE}^{3}$. De nuevo, total de población y su distribución según lugar de nacimiento (España o extranjero) son las variables de las que disponemos, en este caso para los 364 núcleos y 223 diseminados existentes en el conjunto de municipios malagueños. Posteriormente, en ambos casos (seccionado y Nomenclátor) hemos seleccionado los municipios rurales, esto es, los menores de 10.000 habitantes, un total de 84 , con poblaciones que oscilan entre los 9.118 del mayor, Pizarra, y los 169 del más pequeño, Atajate.

Siendo imprescindible la información estadística, en cualquier investigación que contemple la vertiente territorial de un fenómeno también es imprescindible contar con una base cartográfica a la que adscribirla. Es por ello que hemos debido obtener la cartografía correspondiente a la Estadística del Padrón Continuo y aquella otra referida al Nomenclátor. En primer lugar, hemos obtenido el seccionado censal de la provincia de Málaga correspondiente a 2018, por petición especial al INE, realizando, a partir de ella, la georreferenciación espacial de la información estadística padronal disponible con esta misma resolución espacial, y a la que hemos hecho referencia en el párrafo anterior. Por su parte, la información cartográfica correspondiente al Nomenclátor ha sido obtenida de los Datos Espaciales de Referencia de Andalucía (IECA, 2019), acudiendo al Grupo Temático 7 (Sistema Urbano): en concreto hemos empleado las capas relativas al poblamiento (capas su03:1, poblamiento entidad colectiva; su03:2, poblamiento entidad singular, y su03:3, poblamiento núcleo diseminado). Como bien indica el IECA, los resultados son una primera versión cartográfica del Nomenclátor de Unidades Poblacionales, y, son fuente fundamental a la hora de encarar cualquier estudio sobre la distribución intramunicipal de la población en municipios que cuentan con una única sección censal. Y, una vez más, hemos procedido a georreferenciar a partir de estas capas cartográficas la información estadística correspondiente al Nomenclátor.

Haciendo una recapitulación, podemos señalar que para dar cumplimiento a nuestro objetivo, hemos trabajado con el volumen correspondiente a 2018 de población total, el número de españoles y el número de extranjeros correspondientes a municipios con volúmenes de población muy reducidos pertenecientes a la provincia de Málaga, obtenidos del Padrón Municipal de Habitantes, empleando dos unidades espaciales distintas: por un lado, el seccionado censal; por otro, la cartografía correspondiente al Nomenclátor.

${ }^{3}$ Puede obtenerse esta misma información detallada, sin coste alguno, a escala de Entidad de Población en el denominado Sistema de Información Demográfica del Padrón, en www.ine.es. Sin embargo, tan sólo cubre, en el momento de escribir estas líneas, el periodo 2003/2014, por lo que para años posteriores debe solicitarse un procesamiento especial, como ha sido nuestro caso. 


\section{SECCIONADO CENSAL VS NOMENCLÁTOR DE ENTIDADES Y NÚCLEOS DE POBLACIÓN EN LOS MUNICIPIOS RURALES.}

Son cuatro ejemplos, correspondientes a cuatro realidades diferentes, los que vamos a emplear para mostrar las ventajas que el uso del Nomenclátor tiene sobre el seccionado censal cuando se trata de estudiar la distribución intramunicipal de la población, ya sea en su conjunto o diferenciada por lugar de nacimiento.

El primero de ellos se centra en la mera descripción de la distribución de la población en el interior del municipio, tomando como caso de estudio Alcaucín. Situado en la comarca de la Axarquía, su población total en 2018 ascendía a tan sólo 2.239 habitantes, por lo que, a partir de los volúmenes poblacionales mínimos y máximos de las secciones censales a los que hicimos referencia, cuenta con una única sección, que equivale a la totalidad del término municipal (figura 2). En consecuencia, el empleo de esta unidad espacial no nos permite conocer cómo se distribuye la población en el interior del municipio, un hecho cuya importancia disminuiría si pudiésemos estar razonablemente seguros de que la práctica totalidad de los empadronados residen en la cabecera municipal. Sin embargo, el caso de Alcaucín no se corresponde con esta situación. Efectivamente, el Nomenclátor identifica en el interior del municipio ocho entidades singulares, de las cuales, además, siete diferencian a su vez entre núcleo y diseminado. Contamos, por tanto, con los 2.239 habitantes del municipio divididos en un total de 15 unidades espaciales diferentes (figura 2), con volúmenes de empadronados que oscilan entre los 505 del núcleo cabecera y los 8 del núcleo de Las Majadas (tabla 2). 
Figura 2. Localización del municipio de Alcaucín, cartografía correspondiente al seccionado censal y cartografía correspondiente al Nomenclátor (2018).
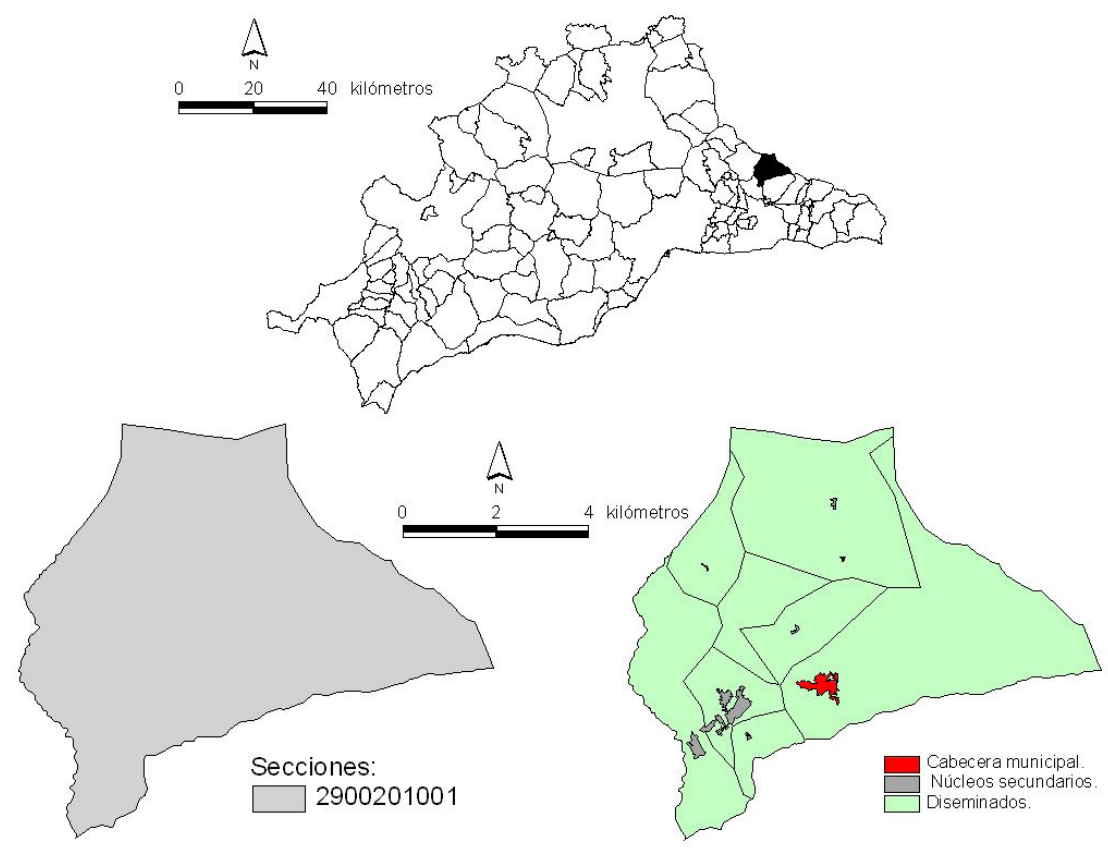

Fuente: Datos Espaciales de Referencia de Andalucía, IECA. Elaboración propia.

Tabla 2. Municipio de Alcaucín. Población en núcleos y diseminados (2018).

\begin{tabular}{|l|l|l|}
\hline Código & Denominación & Total \\
\hline 29002000101 & ALCAUCÍN & 505 \\
\hline 29002000199 & DISEMINADO & 265 \\
\hline 29002000201 & CERRO (EL) & 17 \\
\hline 29002000301 & $\begin{array}{l}\text { CORTIJILLOS } \\
\text { (LOS) }\end{array}$ & 27 \\
\hline 29002000399 & DISEMINADO & 96 \\
\hline 29002000401 & ESPINO & 43 \\
\hline 29002000402 & MAJADAS (LAS) & 8 \\
\hline
\end{tabular}




\begin{tabular}{|l|l|l|}
\hline Código & Denominación & Total \\
\hline 29002000499 & DISEMINADO & 65 \\
\hline 29002000599 & DISEMINADO & 150 \\
\hline 29002000602 & PUENTE (EL) & 545 \\
\hline 29002000699 & DISEMINADO & 126 \\
\hline 29002000701 & TORIL (EL) & 19 \\
\hline 29002000799 & DISEMINADO & 22 \\
\hline 29002000801 & VENTA BAJA & 214 \\
\hline 29002000899 & DISEMINADO & 137 \\
\hline
\end{tabular}

Fuente: Nomenclátor de Población. Instituto Nacional de Estadística.

La ventaja del empleo del Nomenclátor es evidente; como además disponemos de esta misma información para diferentes años, puede encararse también un estudio sobre la evolución de la distribución de la población en el interior del municipio, estudio imposible de abordar acudiendo al seccionado.

Por otro lado, anteriormente se había indicado que la presencia de inmigrantes extranjeros retirados tenía cierta importancia en determinados municipios de la provincia malagueña, muchos de ellos rurales, por lo que, además de conocer cómo se distribuye el total de la población en su interior, puede ser también de utilidad prestar atención a cómo se distribuye en función de su origen. Para ejemplificar esto nos centraremos en el caso de Sedella. Situado, como Alcaucín, en la comarca de la Axarquía, este pequeño municipio de 606 habitantes en 2018 contaba con un porcentaje de nacidos en el extranjero del $35,3 \%$; un valor muy por encima del correspondiente a la provincia $(18,4 \%)$, a la Comunidad Autónoma $(9,4 \%)$ y al conjunto de España $(13,7 \%)$.

De nuevo el municipio cuenta con una única sección censal (figura 3), por lo que a partir de aquí no es posible conocer cómo se distribuye la población en su interior, menos aun diferenciándola por lugar de nacimiento; así las cosas, de optar por el seccionado como unidad espacial de referencia tan sólo podríamos indicar que su volumen de población es reducido, y que el porcentaje de nacidos en el extranjero es muy relevante. Sin embargo, acudir al Nomenclátor nos permite afinar bastante más en la cuestión de la distribución espacial según su origen, y aportar evidencias estadísticas de un fenómeno bastante común en la comarca malagueña de la Axarquía: la presencia de importantes volúmenes de población extranjera afincada en los diseminados municipales (Junta, 2006). 


\section{Figura 3. Localización Del Municipio De Sedella, Cartografía Correspondiente Al} Seccionado Censal Y Cartografía Correspondiente Al Nomenclátor (2018).
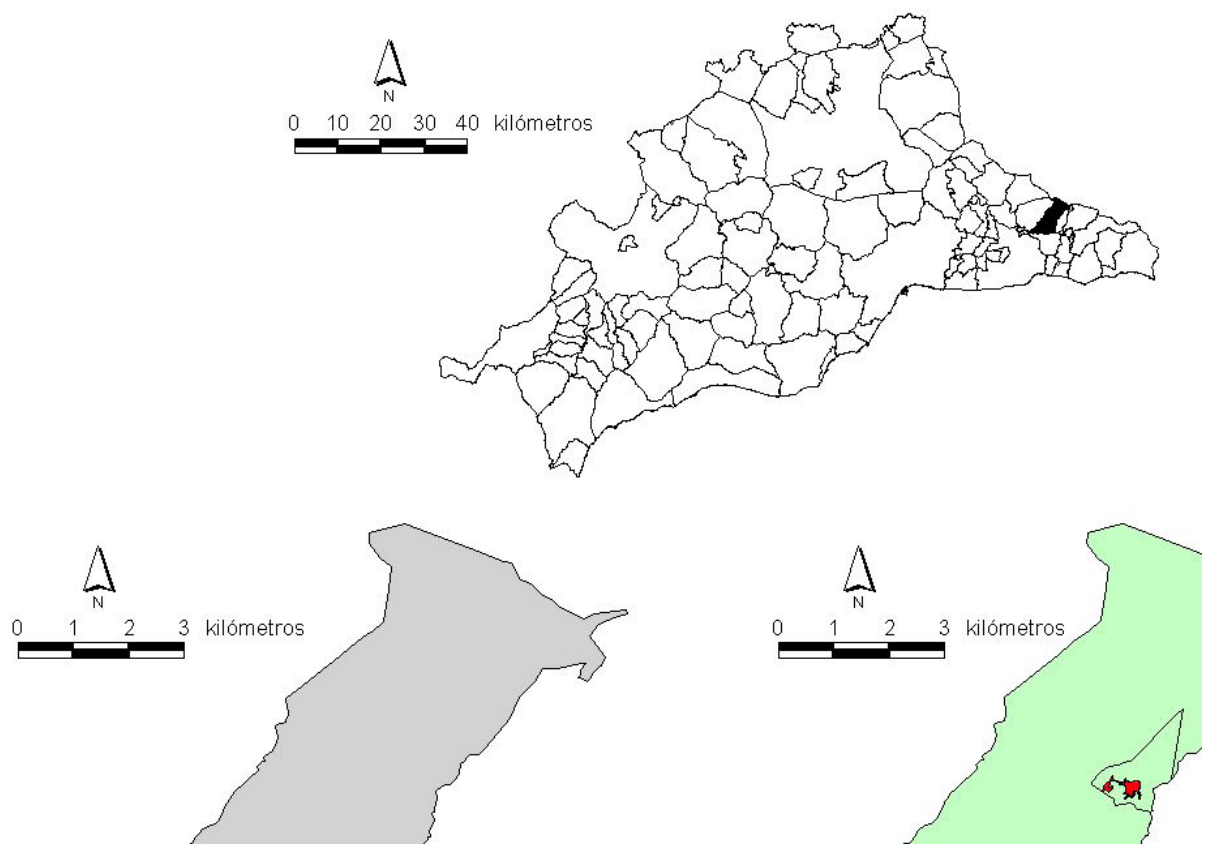

Fuente: Datos Espaciales de Referencia de Andalucía, IECA. Elaboración propia.

Una vez más el Nomenclátor identifica más unidades espaciales que el seccionado (tabla 3): en concreto dos núcleos, Sedella, la cabecera municipal, y Rubite, un pequeño núcleo localizado en el extremo sudoccidental del municipio, con un diseminado asociado al mismo (figura 3). Además, el Nomenclátor considera al resto del término municipal como diseminado, no adscrito a núcleo alguno, en la tabla 3 con el código 29087000399. Con esta información podemos comprobar cómo dos terceras partes de los empadronados del municipio residen en su cabecera, que en Rubite y su diseminado lo hace un total de 30 personas, y que nada menos que el $29 \%$ de la población reside en viviendas dispersas por el resto del término municipal. Una información sobre la distribución de la población imposible de obtener empleando el seccionado censal. 
Tabla 3. Municipio de Sedella. Población en núcleos y diseminados, valor total y según lugar de nacimiento (2018).

\begin{tabular}{|l|l|l|l|l|}
\hline Código & Denominación & Total & España & Extranjero \\
\hline 29087000101 & RUBITE & 12 & 6 & 6 \\
\hline 29087000199 & DISEMINADO & 18 & 9 & 9 \\
\hline 29087000201 & SEDELLA & 399 & 321 & 78 \\
\hline 29087000399 & DISEMINADO & 177 & 56 & 121 \\
\hline
\end{tabular}

Fuente: Nomenclátor de Población. Instituto Nacional de Estadística.

Pero es más, si distribuimos estos contingentes de población según su lugar de nacimiento (tabla 3), podemos comprobar cómo efectivamente el grueso de los nacidos en el extranjero residen en diseminado, población que, además, es a su vez el grueso de los residentes en este tipo de hábitat. Mientras que, al contrario, el grueso de los nacidos en España reside en núcleo -básicamente en la cabecera municipal-, contingente que, a su vez, es el grueso de los residentes en este tipo de hábitat. Con ello, resulta evidente que el empleo del Nomenclátor nos permite aportar evidencias cuantificables de que, en gran medida, el diseminado, en el caso de Sedella, está habitado básicamente por población de origen extranjero. Este es el soporte estadístico al que en los párrafos anteriores hacíamos referencia, y que sólo es accesible desde el Nomenclátor.

Esta misma situación es extrapolable a municipios con mayores volúmenes de población, en los que, en virtud de ellos, existen dos secciones censales. Tomemos el caso de Behanavís. Se trata de un municipio, situado en el trasfondo montañoso de la Costa del Sol Occidental, que en 2018 contaba con un total de 7.900 empadronados, divididos en dos secciones censales (tabla 4), unas secciones que, como se aprecia en la tabla, superan ampliamente los valores máximos de población a los que venimos haciendo referencia. Podemos aventurar que esta situación se deriva del elevado volumen de población extranjera presente en ellas, amplia mayoría. Esta población extranjera no debe ser considerada como "población electora" en su totalidad (los requisitos para poder ejercer el derecho a voto en las elecciones municipales implica que el $73 \%$ de los extranjeros que residen legalmente en España no puedan ejercer ese derecho -Gutiérrez y Ordaz, 2019), por lo que, estrictamente, no entran en el cómputo de la población electora residente en la sección. Tengamos en cuenta que lo que, según la LOREG, define los límites de población de la sección es el número de electores, no el volumen total de empadronados. 
Tabla 4. Municipio de Benahavís. Distribución de la población por sección censal y lugar de nacimiento (2018).

\begin{tabular}{|l|l|l|l|}
\hline & Total Población & España & Extranjero \\
\hline 2902301001 & 4.702 & 1.922 & 2.780 \\
\hline 2902301002 & 3.287 & 947 & 2.340 \\
\hline $\begin{array}{l}\text { Total munici- } \\
\text { pal }\end{array}$ & 7.989 & 2.869 & 5.120 \\
\hline
\end{tabular}

Fuente: Estadística del Padrón Continuo. Instituto Nacional de Estadística.

La cartografía del seccionado se muestra en la figura 4, y a partir de ella se puede derivar el hecho de que es la porción sudoriental del municipio la más densamente poblada -a partir de la población de las secciones y su extensión espacial-, y que es en esta misma porción -correspondiente a la sección 002donde la presencia de población extranjera es proporcionalmente superior.

Figura 4. Localización del municipio de Benahavís, cartografía correspondiente al seccionado censal y cartografía correspondiente al Nomenclátor (2018).

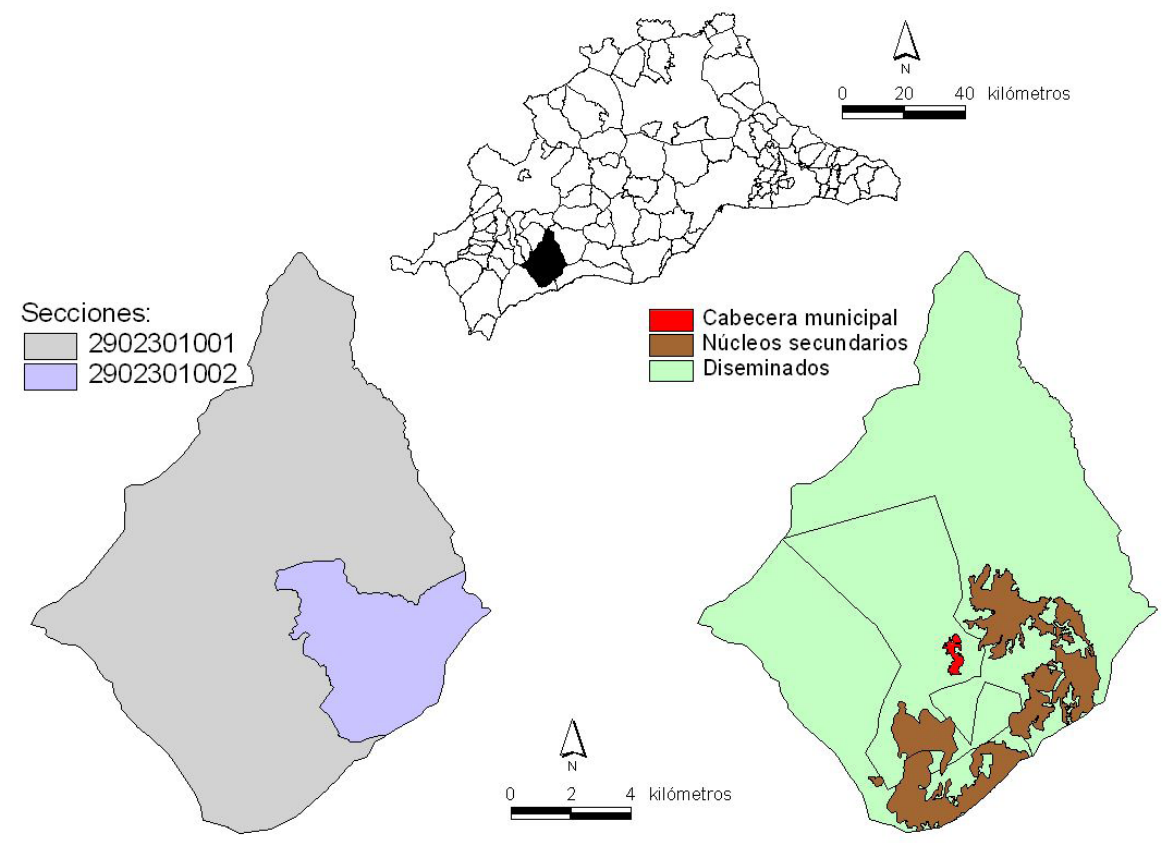

Fuente: Datos Espaciales de Referencia de Andalucía, IECA. Elaboración propia. 
Sin embargo, una vez más el empleo del Nomenclátor permitiría enriquecer muy notablemente la descripción de la distribución de la población en Benahavís. En efecto, esta fuente adscribe los 7.989 empadronados a un total de 9 unidades espaciales, 6 núcleos y 3 diseminados (tabla 5); pudiéndose observar cómo la cabecera municipal es la única en la que los nacidos en España son mayoría -muy ligera, de todas formas-, al tiempo que Artola, en el límite oriental del municipio y colindante con las urbanizaciones de lujo de Nueva Andalucía, en Marbella, no sólo concentra más población que la propia cabecera, sino que, además, es muy mayoritariamente nacida en el extranjero. A partir de la información contenida en la tabla podría ahondarse considerablemente más en las características de la distribución espacial de la población que empleando el seccionado censal.

Tabla 5. Municipio de Benahavís. Población en núcleos y diseminados, valor total y según lugar de nacimiento (2018).

\begin{tabular}{|l|l|l|l|l|}
\hline Código & Denominación & Total & $\begin{array}{l}\text { Españo- } \\
\text { les }\end{array}$ & $\begin{array}{l}\text { Extranje- } \\
\text { ros }\end{array}$ \\
\hline 29023000101 & ARTOLA & 3.037 & 862 & 2.167 \\
\hline 29023000104 & ZABALETA (LA) & 9 & 0 & 9 \\
\hline 29023000199 & DISEMINADO & 241 & 85 & 154 \\
\hline 29023000201 & BENAHAVÍS & 2.357 & 1.238 & 1.115 \\
\hline 29023000202 & MONTEMAYOR & 27 & 6 & 21 \\
\hline 29023000299 & DISEMINADO & 135 & 95 & 40 \\
\hline 29023000302 & $\begin{array}{l}\text { ALQUERÍA-EL PA- } \\
\text { RAÎSO (LA) }\end{array}$ & 971 & 230 & 741 \\
\hline 29023000303 & $\begin{array}{l}\text { PARQUE BOTÁNI- } \\
\text { CO }\end{array}$ & 2 & 1 & 1 \\
\hline 29023000399 & DISEMINADO & 1.210 & 352 & 856 \\
\hline
\end{tabular}

Fuente: Nomenclátor de Población. Instituto Nacional de Estadística.

Otra situación es aquella en la que la delimitación de las secciones en el interior del municipio distorsiona las interpretaciones que, a partir de ella, pudieran hacerse sobre la distribución de la población en el municipio. Se trata, por tanto, de una variante del conocido problema del "tablero de ajedrez", que aqueja a los cálculos de los índices de diferenciación residencial urbana (Openshaw, 1984; Linares, 2012). En este caso se tomará como ejemplo el municipio de Ojén; sus 
3.568 empadronados se encuadran en dos secciones censales, la 297601001 y la 297601002 (tabla 6). La primera de ellas se extiende por la mitad oriental del municipio (figura 5), y cuenta con un total de 2.455 empadronados, mientras que la segunda lo hace por la mitad occidental, estando empadronados en ella 1.113 residentes. Junto a los volúmenes totales de población también ofrecemos la distribución de la población según su lugar de nacimiento, y la inspección de las cifras nos indica que el grueso de los nacidos en el extranjero reside en la sección oriental (sección 001), siendo franca minoría (menos del 15\%) en la occidental (sección 002).

Tabla 6. Distribución de la población por sección censal según lugar de nacimiento (España/Extranjero). Municipio de Ojén, 2018.

\begin{tabular}{|l|l|l|l|}
\hline Sección & $\begin{array}{l}\text { Total Pobla- } \\
\text { ción }\end{array}$ & $\begin{array}{l}\text { Espa- } \\
\text { ña }\end{array}$ & Extranjero \\
\hline 2907601001 & 2.455 & 1.768 & 687 \\
\hline 2907601002 & 1.113 & 952 & 161 \\
\hline Total población & 3.568 & 2.720 & 848 \\
\hline
\end{tabular}

Fuente: Estadística del Padrón Continuo. Instituto Nacional de Estadística. 
Figura 5. Localización del municipio de Ojén, cartografía correspondiente al seccionado censal y cartografía correspondiente al Nomenclátor (2018).
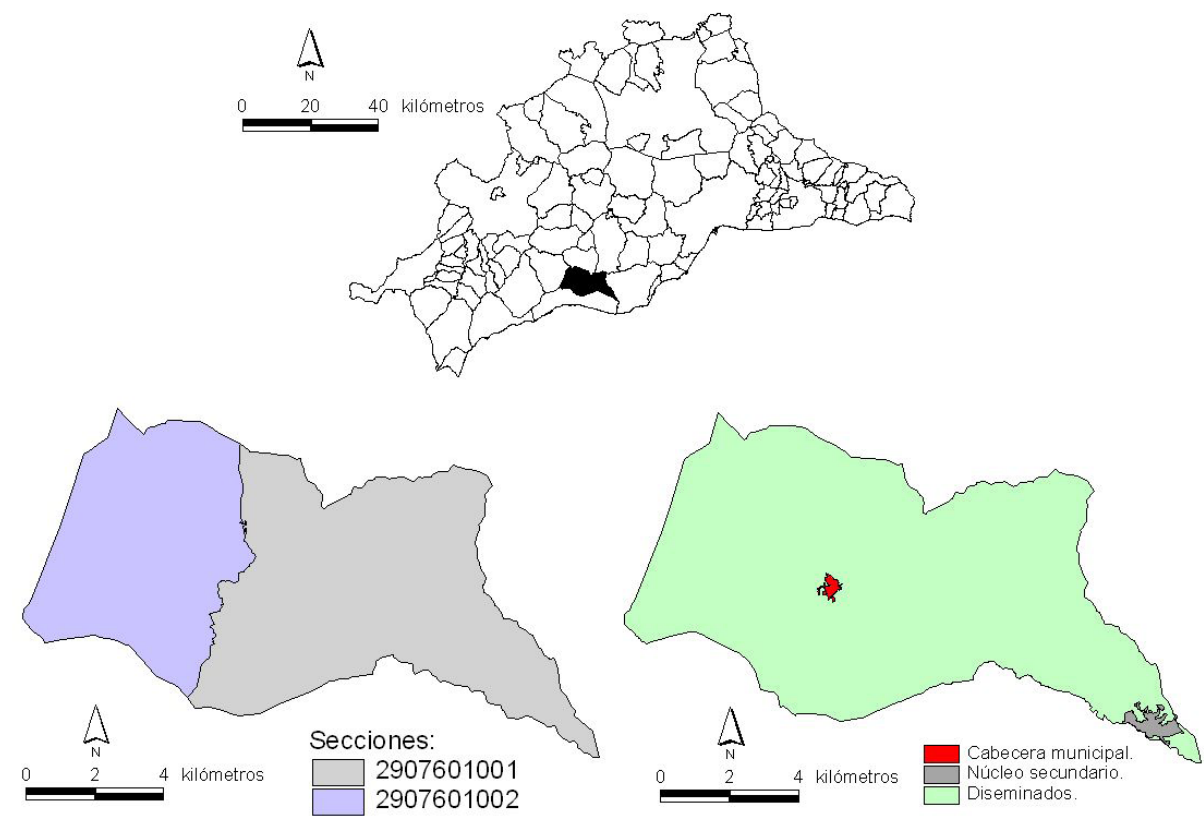

Fuente: Datos Espaciales de Referencia de Andalucía, IECA. Elaboración propia.

Por su parte, el Nomenclátor identifica en el municipio dos núcleos: la cabecera municipal, y otro, de menor población, en el extremo sudoccidental (figura 5), quedando el resto del término adscrito a un diseminado genérico, tal y como se aprecia en la tabla 7. En este momento lo que nos interesa es la cartografía asociada al Nomenclátor, puesto que, como inmediatamente se verá, permite matizar sobremanera la distribución espacial de la población según su origen que se deriva del empleo del seccionado censal. 
Tabla 7. Distribución de la población por Entidades, núcleos y diseminados de la población según lugar de nacimiento (España/Extranjero). Municipio de Ojén, 2018.

\begin{tabular}{|l|l|l|l|l|}
\hline Código & Denominación & Total & Españoles & Extranjeros \\
\hline 29076000101 & OJÉN & 2.849 & 2.379 & 468 \\
\hline 29076000102 & MAIRENA (LA) & 404 & 120 & 284 \\
\hline 29076000199 & DISEMINADO & 315 & 221 & 94 \\
\hline
\end{tabular}

Fuente: Nomenclátor de Población. Instituto Nacional de Estadística.

Efectivamente, el Nomenclátor indica que en el primero de los núcleos, la villa de Ojén, se concentra el grueso de la población, siendo muy mayoritarios los residentes españoles. Sin embargo, la situación del núcleo de La Mairena es la contraria: en él son amplia mayoría (dos terceras partes) los empadronados nacidos en el extranjero. Como puede apreciarse en la figura 5 La Mairena está situada en el extremo sudoccidental del municipio, lindando con áreas urbanas caracterizadas por altos niveles de calidad del municipio de Marbella (El Rosario, Los Monteros, Elviria), niveles que este núcleo comparte con ellos. Pero lo destacable es que el análisis de la información del nomenclátor nos permite identificar a La Mairena como un "enclave" de población mayoritariamente nacida en el extranjero, en el contexto de un municipio en el que la población española es muy mayoritaria tanto en el otro núcleo como en el diseminado. Es más, el seccionado censal divide a la villa de Ojén en dos, la mitad septentrional incluida en la sección segunda, la mitad meridional en la sección primera, lo que enmascara aún más la verdadera distribución de la población, y, en consecuencia, hace aún menos aconsejable el empleo de esta unidad espacial.

Para terminar, es preciso indicar que incluso en el caso en el que el Nomenclátor identifica una única entidad singular en el municipio, como en su momento indicamos, ésta queda dividida en núcleo y, si existe población en esa condición, en diseminado. Y, una vez más, es posible realizar un estudio mucho más exhaustivo de los residentes a partir del Nomenclátor que empleando el seccionado censal. Para ejemplificar esto, nos centraremos en Cómpeta, situado en la comarca de la Axarquía, con un total de 3.745 empadronados, de los cuales 2.061 son españoles y el resto extranjeros. Se trata de un municipio en el que la presencia es estos últimos es porcentualmente muy elevada (suponen el 45\%), y utilizando el seccionado censal poco más podríamos decir. Sin embargo, la información que nos ofrece el Nomenclátor permite ahondar en la distribución territorial de ambos grupos de población, tal y como se aprecia en la tabla 8. 
Tabla 8. Distribución de la población por Entidades, núcleos y diseminados de la población según lugar de nacimiento (España/Extranjero). Municipio de Cómpeta, 2018.

\begin{tabular}{|l|l|l|l|l|}
\hline Código & Denominación & Total & España & Extranjero \\
\hline 29045000101 & CÓMPETA & 2.471 & 1.884 & 587 \\
\hline 29045000199 & DISEMINADO & 1.274 & 177 & 1.097 \\
\hline
\end{tabular}

Fuente: Nomenclátor de Población. Instituto Nacional de Estadística.

Efectivamente, a partir de esta fuente se puede comprobar cómo un tercio de los empadronados residen en viviendas dispersas por el conjunto del término municipal. Y también se pone de manifiesto cómo las pautas de distribución de la población varían en función de su lugar de nacimiento: los nacidos en España tienden a residir de forma muy mayoritaria en la cabecera del municipio, mientras que la pauta correspondiente a los nacidos en el extranjero es justamente la contraria: el $86 \%$ de ellos residen en diseminado, tipo de hábitat en el que, además, se resuelven como las tres cuartas partes del total. No sólo estaríamos añadiendo evidencia estadística a la comentada presencia mayoritaria de extranjeros en los diseminados axárquicos, sino que, distribuyendo esta población por grupos de edad -información que también está disponible a este nivel de resolución espacial- podríamos ahondar en la caracterización de la población según su lugar de residencia.

\section{CONCLUSIONES}

El estudio de la población a partir de fuentes censales o padronales presenta dificultades, derivadas en el primer caso de la metodología muestral, de la escasa amplitud temática en el segundo. Pero estas dificultades se acrecientan cuando la investigación se realiza a escala inframunicipal, debido a que el empleo de la unidad espacial de referencia más al uso, la sección censal, contempla una única sección en los municipios con contingentes de población pequeños, que equivale a la totalidad del término municipal. En estos casos es imposible acceder a cómo se distribuyen los empadronados en el interior del municipio en el que residen. Existe, sin embargo, otra delimitación espacial a la que queda referida la información padronal, el Nomenclátor de Entidades y Núcleos de Población, que identifica la totalidad de núcleos y diseminados existentes en cada uno de los municipios. En las páginas anteriores se han mostrado las ventajas que tiene su empleo, no sólo cuando el Nomenclátor identifica más unidades espaciales que el seccionado, sino también cuando identifica un único núcleo, al considerarse el resto del término municipal como diseminado, añadiendo, de facto, una segunda 
delimitación espacial. Es más, en los casos en los que esta fuente indica que la totalidad de la población reside en un único núcleo, sin presencia alguna de población en diseminado, estimamos que, aunque la presentación de las cifras sería equivalente en el seccionado - toda la población adscrita a una única sección- y en el Nomenclátor -toda la población adscrita a un único núcleo-, desde el punto de vista de la representación cartográfica la correspondiente al segundo es superior, pues mostraría a los empadronados concentrados en la cabecera municipal, quedando el resto del término vacío. Es el caso del pequeño municipio de Atajate, con tan sólo 169 habitantes, todos concentrados en la cabecera.

Además, también se ha mostrado cómo el uso de la cartografía procedente del Nomenclátor permite soslayar un problema más propio de los ámbitos urbanos, el del tablero de ajedrez. La interpretación de la distribución de la población en el caso de Ojén se distorsiona si se emplea el seccionado, puesto que la cabecera queda dividida entre las dos secciones del municipio. Un problema que desaparece si nos decantamos por el Nomenclátor.

Con todo ello creemos que hemos mostrado cómo, efectivamente, el empleo del Nomenclátor presenta indudables ventajas sobre el seccionado censal en contextos rurales. Sin embargo, y siendo esto así acudir a esta fuente en entornos urbanos no nos parece aconsejable, puesto que en ellos el seccionado censal divide el término municipal en un mayor número de unidades territoriales. A modo de ejemplo, el municipio de Marbella, con 141.463 empadronados en 2018, cuenta con 76 secciones distribuidas en 4 distritos, por tan solo 12 divisiones en el Nomenclátor. Es más, para determinados individuos urbanos disponemos, como en su momento indicamos, de una unidad especial de referencia más, el barrio, para el que está disponible también la información padronal. Ésta es una unidad espacial con significado sociológico, y, allí donde está disponible junto con información estadística asociada (Málaga, Granada y Sevilla son ejemplos en Andalucía), en nuestra opinion es la mejor opción. Pero lógicamente es el investigador el que debe decidir sobre su empleo, a la vista de sus objetivos y de la información estadística con la que realmente cuenta.

\section{BIBLIOGRAFÍA}

BAYONA, J., y GIL, F. (2013): "Is foreing immigration the solution to rural depopulation?", Sociologia Ruralis, 53 (1), pp. 26-51.

BOLETÍN OFICIAL DEL ESTADO (1985): Ley Orgánica 5/1895, de 19 de junio, del Régimen Electoral General. BOE 147 de 20 de junio de 1985. Madrid, Boletín Oficial del Estado.

BURRIEL, E. (2019): "La larga huella en el territorio de las viviendas secundarias ilegales. El ejemplo de Gilet (Valencia)", Cuadernos de Geografía de la Universitat de Valencia, 102, pp. 107-140.

CAMARERO, L. -coord.- (2009): La población rural de España. De los desequilibrios a la sostenibilidad social, Barcelona, Fundación «La Caixa». 
COLLANTES GUTIÉRREZ, F. (2010): El impacto demográfico de la inmigración en la España rural despoblada. Documento de trabajo 30/2010, Madrid, Real Instituto Elcano.

COLlANTES, F., PINILlA, V., SÁEZ, L. A., y SIlVESTRE, J. (2014): "Reducing depopulation un Rural Spain.: the impact of immigration", Population, Space and Place, 20, pp. 606-621.

CONSEJO ECONÓMICO Y SOCIAL (2018): El medio rural y su vertebración social y territorial, Madrid, Consejo Económico y Social España.

DEFENSOR DEL PUEBLO (2019): La situación demográfica en España. Efectos y consecuencias. Separata del Volumen II del informe anual 2018, Madrid, Defensor del Pueblo.

EUSTAT INSTITUTO VASCO DE ESTADÍSTICA (sin fecha): Sección Censal, disponible en: http://www.eustat.eus/documentos/opt 0/tema 159/elem 3830/definicion.html [Consulta: 23-9-2019]

GUTIÉRREZ, I., y ORDAZ, A. (5/15/2019): "El 73\% de los extranjeros que viven legalmente en España no tiene derecho al voto". El diario.es, disponible en https://www. eldiario.es/desalambre/voto-inmigrante-extranjeros-legalmenteEspana 0 880512622.html [Consulta: 1-10-2019]

HAGGET, P. (1976): Análisis locacional en la Geografía Humana, Madrid, Gustavo Gili.

HERMOSILLA, J. (1992): "La residencia secundaria en la periferia occidental del área metropolitana de Valencia", Cuadernos de Geografía, 51, pp. 95-109.

INSTITUTO DE ESTADÍSTICA Y CARTOGRAFÍA DE ANDALUCÍA (sin fecha): Nomenclátor de entidades y núcleos de población de Andalucía, disponible en

https://www.juntadeandalucia.es/institutodeestadisticaycartografia/nomenclator/metodologia/metodologia.pdf [Consulta: 24-9-2019].

INSTITUTO DE ESTADÍSTICA Y CARTOGRAFÍA DE ANDALUCÍA (2019): Datos Espaciales de Referencia de Andalucía (DERA), disponible en https://www.juntadeandalucia.es/institutodeestadisticaycartografia/DERA/ [Consulta: 30-9-2019]

INSTITUTO NACIONAL DE ESTADÍSTICA (2011): Informe metodológico estandarizado: Censo de Población, disponible en:

http://www.ine.es/dynt3/metadatos/es/RespuestaDatos.htm?oe=30243 [Consulta 29-92019]

JUNTA DE ANDALUCÍA (2006): Plan de Ordenación del Territorio de la Costa del Sol Oriental Axarquía. Memoria informativa, Sevilla, Junta de Andalucía.

LINARES, S. (2012): "Dificultades metodológicas al medir la segregación: el problema del tablero de ajedrez y de la unidad espacial modificable", Geografía y Sistemas de Información Geográfica, 4, pp. 10-22.

MONTOSA MUÑOZ, J., y REYES CORREDERA, J. (2017): "El impacto de la gran recesión en el crecimiento urbano de la aglomeración de Málaga", Ería, revista de Geografía, 37(2), pp. 201-216.

NATERA RIVAS, J.J. (1996): “Cambios en el patrón de asentamientos del trasfondo montañoso de la Costa del Sol Occidental en los últimos treinta años", Cuadernos Geográficos de la Universidad de Granada, 26, pp. 223-242.

NATERA RIVAS, J.J., y BATISTA ZAMORA, A.E. (2018): "Flujo y reflujo de la población rural en el periodo 2003/2017. El caso de la Axarquía malagueña", en Nuevas realidades rurales en tiempos de crisis: territorios, actores, procesos y políticas, Granada, Universidad de Granada, pp.196-207. 
NEL-LO, O. (2011): "Estrategias para la contención y gestión de las urbanizaciones de baja densidad en Cataluña", Ciudad y Territorio. Estudios Territoriales, 167, pp. 8198.

OCAÑA OCAÑA, C. (2005): "Microanálisis sociodemográfico de espacios urbanos", Boletín de la Asociación de Geógrafos Españoles, 40, pp. 5-34.

OPENSHAW, S. (1984): The modifiable areal unit problem. Norwich, Geobooks.

RECAÑO, J. (2017): "La sostenibilidad demográfica de la España vacía», Perspectives Demográfiques, 7, pp. 1-4.

RODRÍGUEZ, V., LARDIÉS, R., y RODRÍGUEZ, P. (2010): “ $\quad$ La migración y el registro de los jubilados eurpeos en España”, Área: demografía y población, 20, pp. 1-8.

RUBIO GIL, Á., y PASCUAL BELLIDO, N. E. (2017): «Juventud y empleo rural en España: factor de desarrollo a través del consumo y el turismo sostenible», Revista de Estudios de Juventud, 118, pp. 187-203.

VILLARÍN CLAVERÍA, M.C. y SEGURA CALERO, S. (2015): "Relevancia de la sección censal como escala de estudio. Caso práctico del consumo doméstico de agua en el municipio de Sevilla", en Análisis espacial y representación geográfica: innovación y aplicación. Zaragoza, Universidad de Zaragoza, Asociación de Geógrafos Españoles, pp. 433-441. 